\title{
Dose COVID-19 uncovered a new feature of Metronidazole Drug?
}

Mohammad Seyedhamzeh $^{\text {a\# }}$, Bahareh Farasati Far ${ }^{\text {b\# }}$, Mehdi Shafiee Ardestani ${ }^{\text {a* }}$, Shahrzad Javanshir ${ }^{\mathrm{b}}$, Fatmeh Aliabadi ${ }^{\mathrm{c}, \mathrm{d}}$, Hamed Reyhanfard ${ }^{\mathrm{e}}$, Hamidreza Pazoki-Toroudi $^{\mathrm{c}, \mathrm{d}}$

aDepartment of Radio pharmacy, Faculty of Pharmacy, Tehran University of Medical Sciences, Tehran, Iran.

${ }^{\mathrm{b}}$ Heterocyclic Chemistry Research Laboratory, Department of Chemistry, Iran University of Science and Technology, 16846-13114, Tehran, Iran

${ }^{\mathrm{c}}$ Department of Physiology, Faculty of Medicine, Iran University of Medical Sciences, Tehran, Iran

${ }^{d}$ Physiology Research Center, Faculty of Medicine, Iran University of Medical Sciences, Tehran, Iran.

${ }^{\text {e }}$ College of Pharmacy and Health Sciences, Department of Pharmacy Administration and Public Health. St. John's University, NY, 11439, USA.

\#These authors contributed to the work

Correspondence to: Hamidreza Pazoki-Toroudi, Mehdi Shafiee Ardestani

Tel.: +989126383385, +989128267629

E-mail address:

pazokihamidrezatoroudi95@gmail.com,pazoki49@gmail.com

shafieeardestani@gmail.com

\begin{abstract}
:
Studies of coronavirus disease 2019 (COVID-19) as a current global health problem shown the initial plasma levels of most pro-inflammatory cytokines increased during the infection, which leads to patient countless complications. Previous studies also demonstrated that the metronidazole (MTZ) administration reduced related cytokines and improved treatment in patients. However, the effect of this drug on cytokines has not been determined. In the present study, the interaction of MTZ with cytokines was investigated using molecular docking as one of the principal methods in drug discovery and design. According to the obtained results, the IL12-metronidazole complex is more stable than other cytokines, and an increase in the surface and volume leads to prevent to bind to receptors. Moreover, ligand-based virtual screening of several libraries showed metronidazole phosphate, metronidazole benzoate, 1-[1-(2-Hydroxyethyl)-5nitroimidazol-2-yl]-N-methylmethanimine oxide, acyclovir, and tetrahydrobiopterin (THB or BH4) like MTZ by changing the surface and volume prevents binding IL-12 to the receptor. Finally, the inhibition of the active sites of IL-12 occurred by modifying the position of the methyl and hydroxyl functional groups in MTZ.
\end{abstract}


Keywords: COVID-19, cytokine Inhibition, Metronidazole, Interleukin-12, QSAR.

\section{Introduction}

In January 2020, the World Health Organization (WHO) issued a statement declaring the outbreak of the new coronavirus (COVID-19) as the sixth leading cause of public health emergencies worldwide. Coronaviruses (SARS-CoV) are a large group of viruses that can infect animals and humans, which cause respiratory distress [1]. Given a mortality rate of around 3.7\%, compared with a reported influenza death rate of less than $1 \%$, proper care is urgently required. A hyper inflammatory phenomenon, "cytokine storms" appear to play a key role in severe cases of COVID-19. According to the hypothesis mentioned in a Lancet correspondence, some patients suffering from severe forms of COVID-19 are subject to cytokine storm syndrome, and patients who die in intensive care are more likely to experience a disproportionate reaction from their immune system than from the virus itself [2]. Conferring to a recent study, the incubation period of the coronavirus, the duration between the time of contamination and the appearance of the first symptoms, would be five days on average [3]. Infection with new coronavirus (COVID-19) in the early stages is associated with non-specific and general symptoms such as nausea, fatigue, body aches, fever, and dry cough. Several studies have found in patients with a severe form of COVID-19 abnormally high amounts of several cytokines in the blood, including proinflammatory causing lung and multiorgan failure, and eventually death [4]. All patients with COVID-19 should be screened for hyper-inflammation to identify those who would benefit from targeted immunosuppression to prevent acute lung injury [4, 5]. Therefore, by inhibiting the severity of the cytokine storm, immunosuppressant may prevent lung tissue damage and further clinical deterioration.

MTZ is a nitro imidazole group antibiotic with the ability to treat infectious diseases which are used to treat COVID-19 [6]. Gharebaghi et al. showed that administration of this drug to patients with COVID-19 caused to an acceptable reduction in cytokines [7]. However, the key issue in this case is the effect of this drug on the suitable reduction of cytokines. Computational method that provide valuable information in this case are quantitative structure activity relationship (QSAR) and molecular docking studies. Docking is a method widely used for the discovery of new drugs. Docking is a crucial method of predicting the structure of the receptor-ligand complex in discovering alternative medicines and leading compounds and has become more and more prominent. It is possible to determine and describe a complex biomedical event with the presence of these powerful methods [8]. Furthermore, QSAR helps to establish a relationship between structure and activity in drug design [9].

Accordingly, the aim of this study was to investigate and determining how metronidazole, its derivatives and similar compounds affect the activity of cytokines using molecular docking and QSAR methods.

\section{Experimental}




\section{Protein and Ligand structure}

In the present study, the three-dimensional structures of ligand and proteins were obtained from PubChem and PDB database, respectively.

\section{Molecular docking}

The docking of protein-ligand interaction calculations were done by SwissDock and Autodock 4.2 (Figure 1). 3D and geometry optimizations with energy minimization of each molecule were done by density functional theory at B3LYP/631+G $(\mathrm{d}, \mathrm{p})$ level [22-23] implemented using Gaussian 09 program-package. 2D ligand-protein interaction was calculated by Ligplot software [10]. Computed Atlas for Surface Topography of Proteins (CASTp) was used to evaluate the active site, surface, and volume of protein [11]. DRAGON software was used for molecular descriptors calculation [12]. Genetic Algorithm and Partial least squares regression were used for feature selection. Swiss ADME was used to determine the pharmacokinetics properties of molecules [13].

\section{Result and Discussion}

To study the potential inhibitory effect of metronidazole, we detected the molecular docking of MTZ with some cytokines (Table 1). The results were shown in Figure 1. A more negative score indicates MTZ is more likely to dock with which receptor to achieve a more favorable interaction. According to the calculated binding energies, it can be deduced that the interaction between MTZ and IL-12 a more favorable.

Table 1. Abbreviation, PDB Code and estimated $\Delta \mathrm{G}$ for cytokines calculated by autodock software

\begin{tabular}{cccc}
\hline Cytokine & Abbreviation & PDB Code & Estimated $\Delta \mathrm{G} \mathrm{(Kcal/Mol)}$ \\
\hline Interleukin 6 & IL-6 & 4NI9 & -6.18 \\
Interleukin 8 & IL-8 & 5WDZ & -5.92 \\
Interleukin 12 & IL-12 & 1F45 & -6.42 \\
Interleukin 1 beta & IL-1b & 4G6J & -6.34 \\
Tumour Necrosis Factor alpha & TNF-alpha & 4G3Y & -6.01 \\
Interferon gamma & INF-gamma & 1FG9 & -6.27 \\
\hline
\end{tabular}




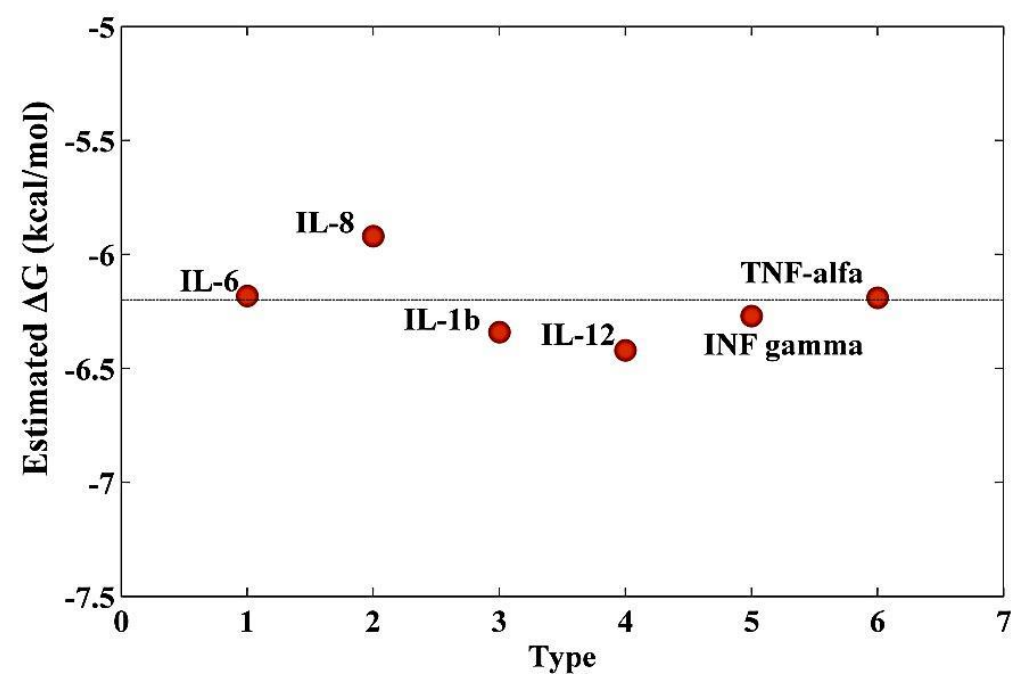

Figure 1. Study of Metronidazole-Cytokine interaction

IL-12 is a heterodimeric compound comprising subunits 35 and $40 \mathrm{kDa}$, which are bind together by a disulfide bond. The primary sources are mononuclear phagocytes and dendritic cells, which act as a potent inducer for Th1 and cause NK proliferation and secretion. Interleukin 12 plays a vital role in increasing Th1 immune and cellular immune responses, and Th1 plays a crucial role in clearing viral infections by secreting INF- $\gamma$. Biological effects are achieved by binding to a specific receptor that increased in secretion, and the up-regulation of interleukin-12 leads to various diseases such as sarcoidosis [14-17].

The binding model of MTZ and IL-12 was shown in Figure 2. The docking results revealed that MTZ and three amino acids, Asn71, Ser241, and Thr242 situated in the binding pocket of IL-12, interact by hydrogen bonding. Also MTZ gets hydrophobic bonds by Ser115 and Pro 178 (Figure 2). In the surface pockets reported by CASTp between structures IL-12, MTZ do not show binding sites for CASTp result showed that compared to did not form a bond by the active site of it (Figure 3), but complex formation caused an increase in area and volume. In other words, MTZ inhibitory ability was altered by increasing in the surface and volume of the particle IL-12 which inhibits to bind IL-12 receptor (Figure 4).

(a) 


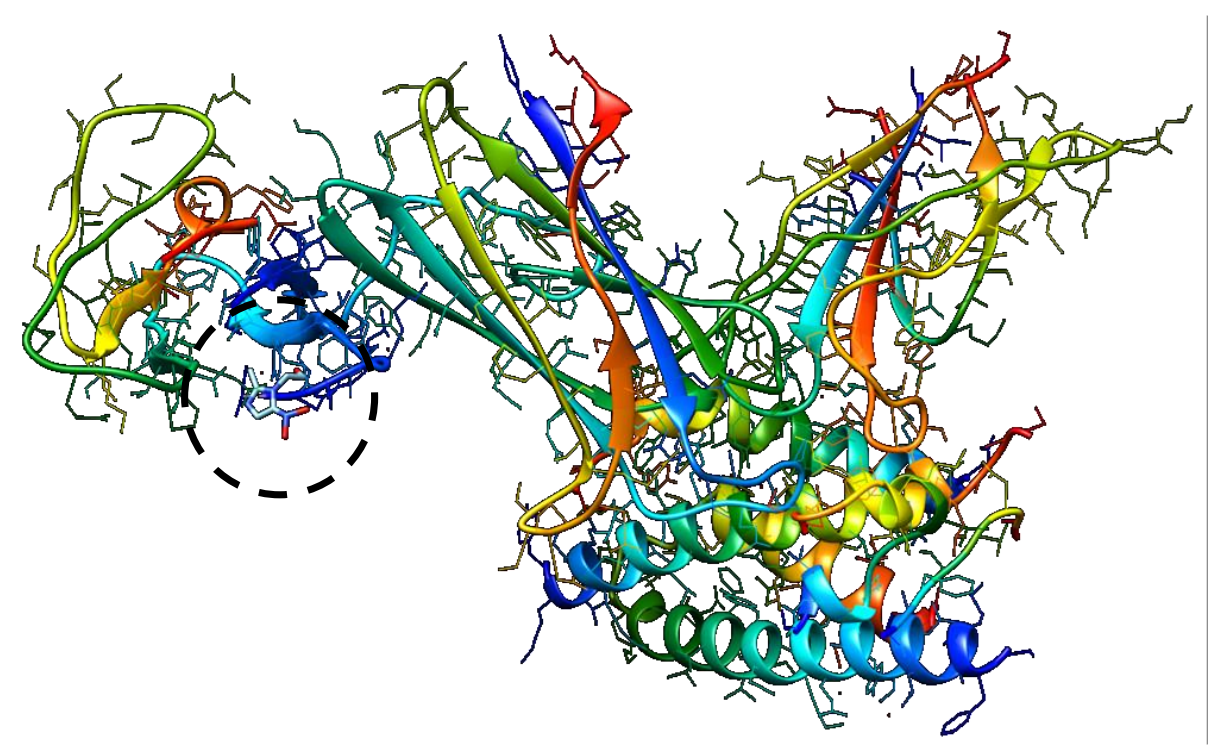

(b)

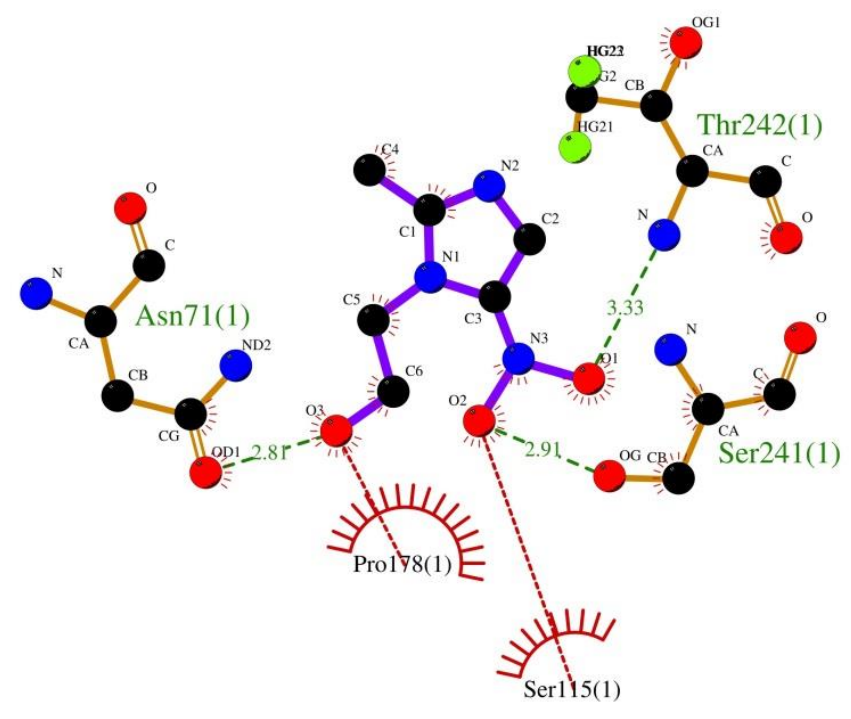

Figure 2. 3D and 2D interaction plot Metronidazole-IL-12 (green line: Hydrogen bond and distance between atoms, Red line Hydrophobic bond) 

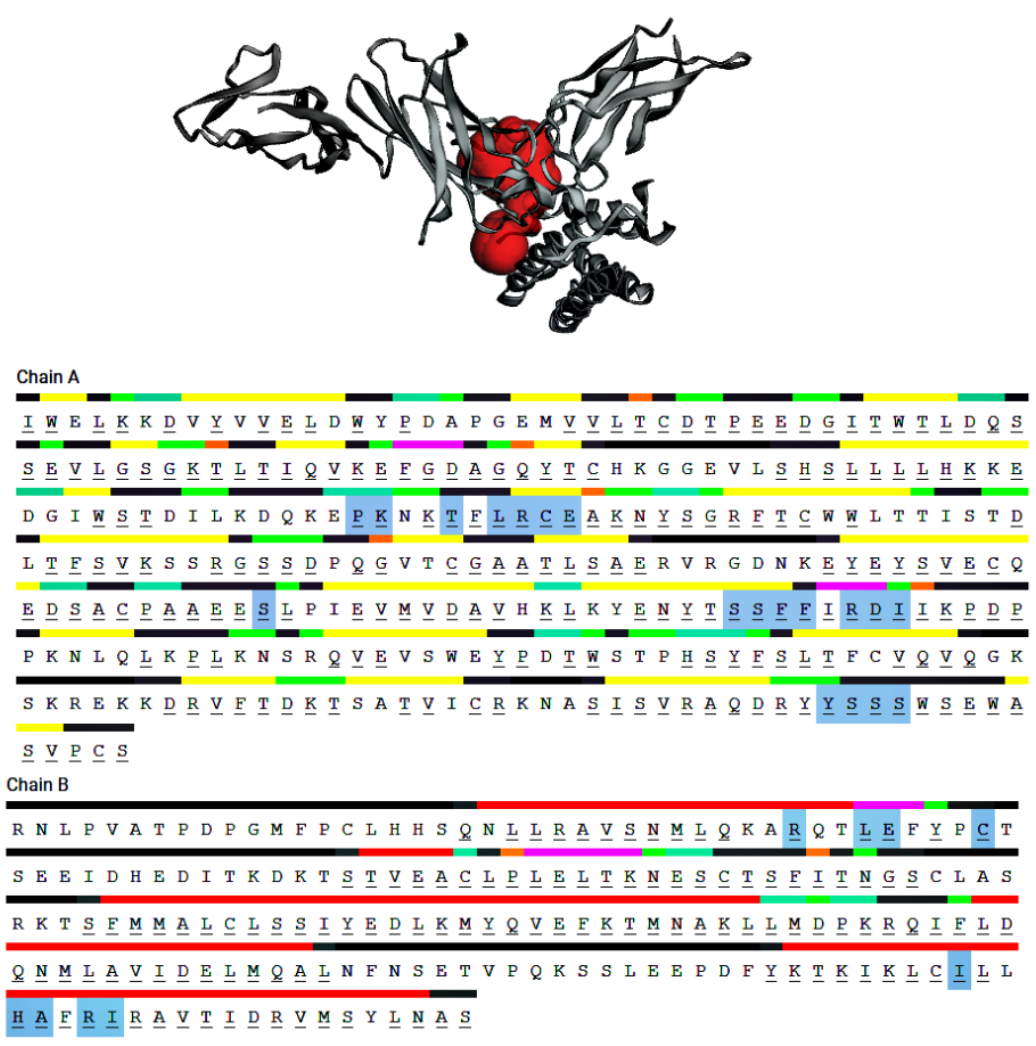

Figure 3. Active cite of IL-12. It marked by blue color

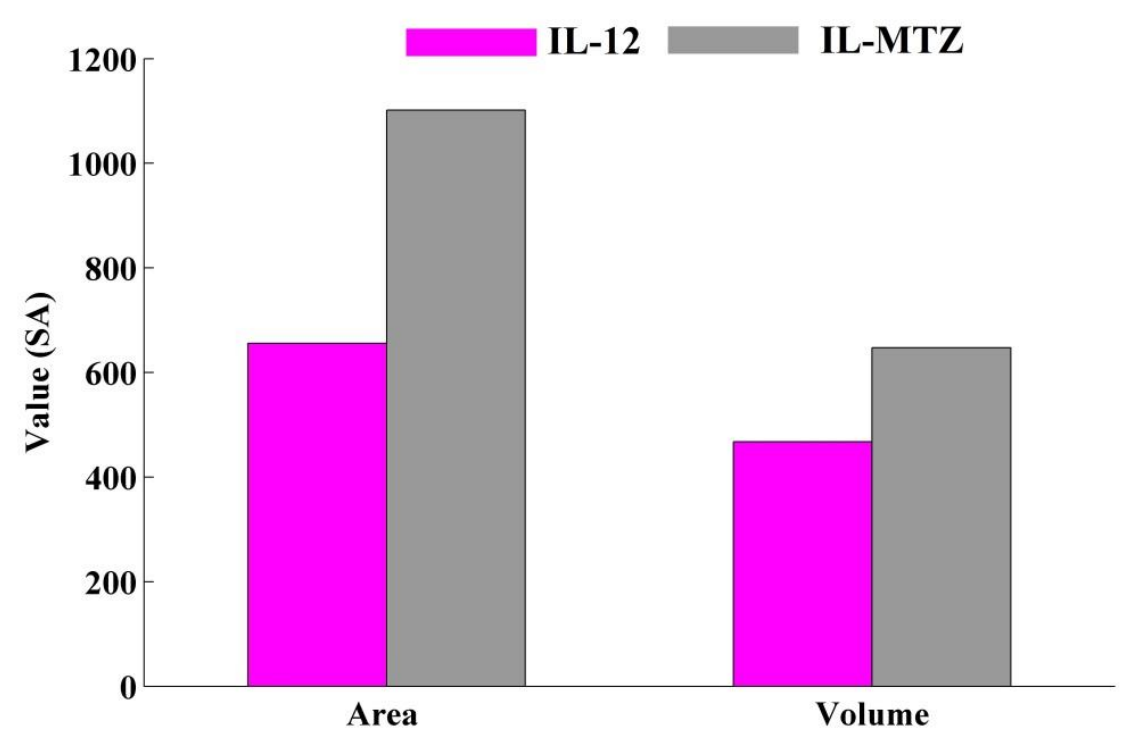

Figure 4. Comparison Area and volume comparison of IL-12 (Pink) and IL-MTZ (Gray)

To compare and evaluate the impact of different substructures in interaction with IL-12 indicated that Metronidazole phosphate (entry 6) and Metronidazole benzoate (entry 7) from FDA- 
approved drug and 1-[1-(2-Hydroxyethyl)-5-nitroimidazol-2-yl]-N-methylmethanimine oxide (entry 13) formed more stable complex. The results of interaction of these materials are shown in the figure 5 and table 2. As can be seen, metronidazole benzoate forms a stable complex only with hydrophobic bonding, whereas metronidazole phosphate forms a complex by through hydrogen bonding, also, 1-[1-(2-Hydroxyethyl)-5-nitroimidazol-2-yl]-N-methylmethanimine oxide gets hydrogen bonds with Lys170 and hydrophobic bonds by Ile131, Tyr40 and Tyr 167 respectively (Table 3). The quantitative structure-activity relationship (QSAR) results in the electromagnetism (MATS2e), topological charge index and the distance between oxygen atoms (in different functional groups) are effective in the stability of these complexes (Figure 6) (Table. 3). Additionally, pharmacokinetics results indicated these compounds have the same properties as metronidazol (Table 4).

Table 2. Measured and predicted $\Delta \mathrm{G}$ for MTZ similar structures. These structures divided to train and test data sets for QSAR study

Entry




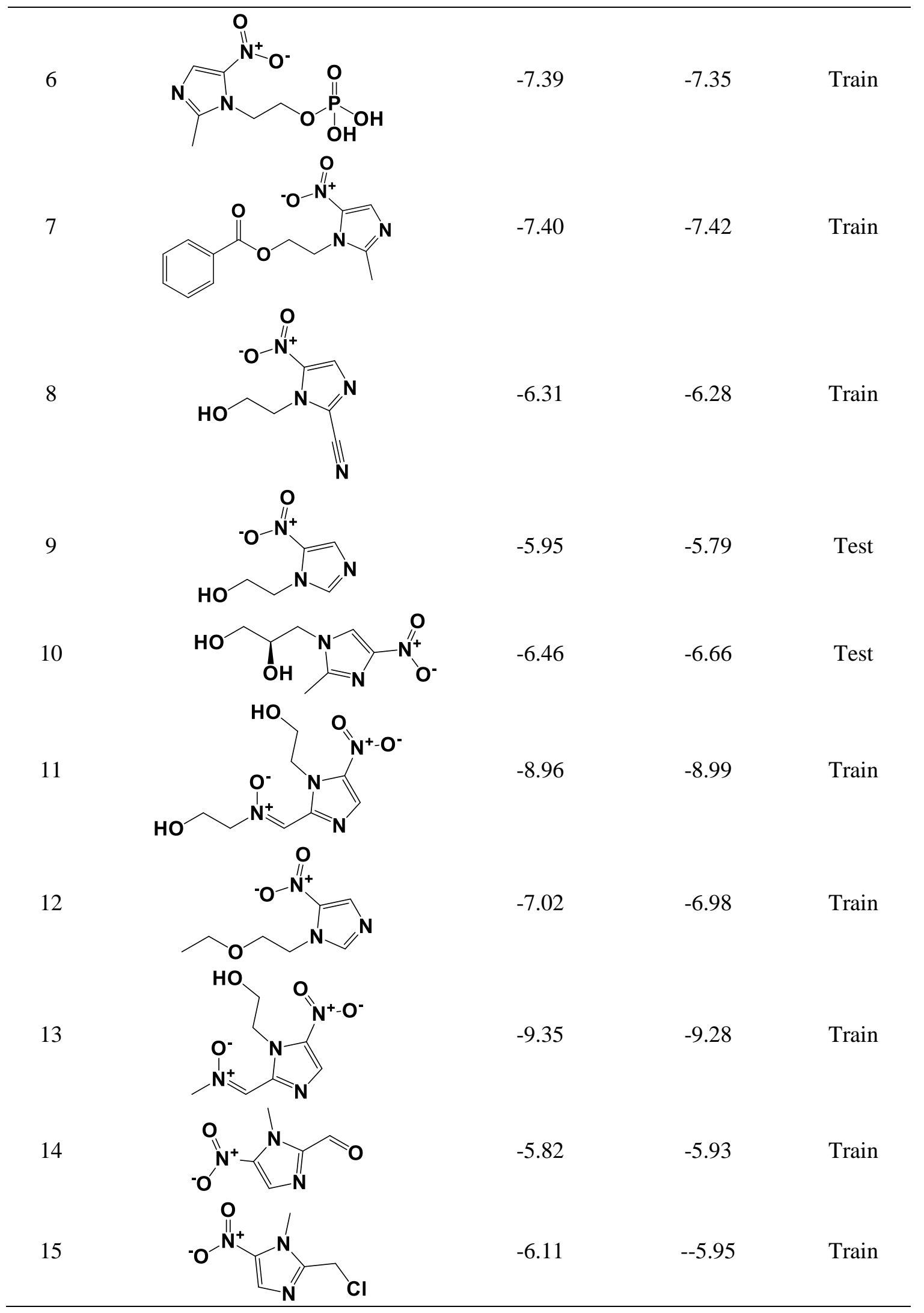




(16

(a)

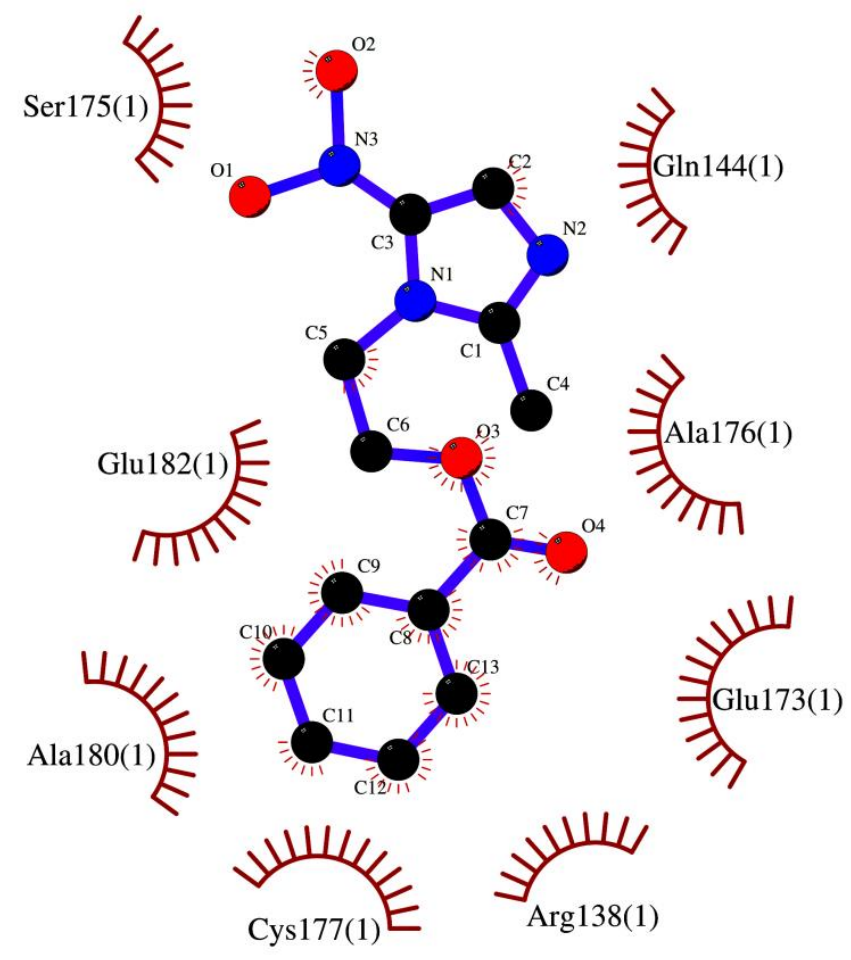

(b) 


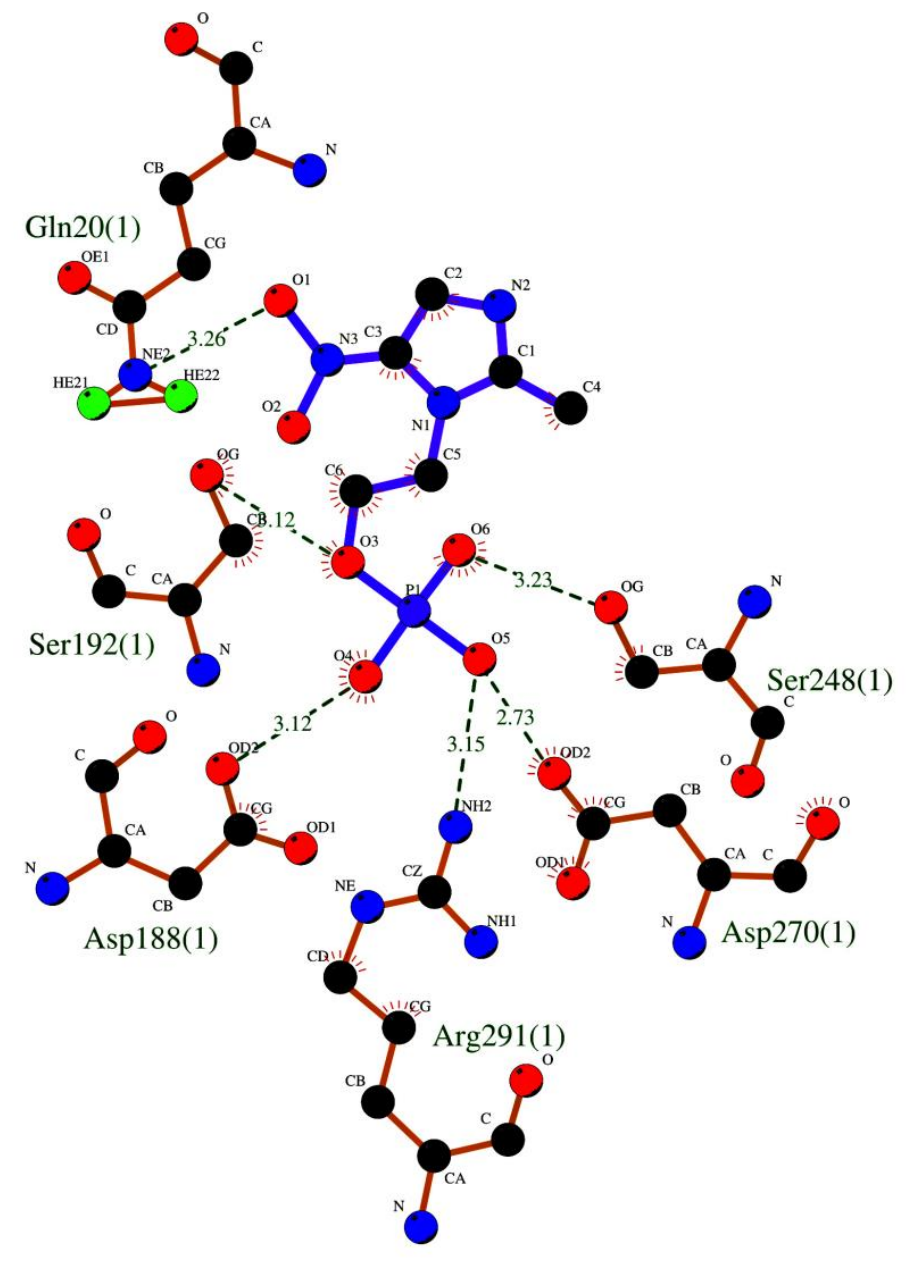

(c) 


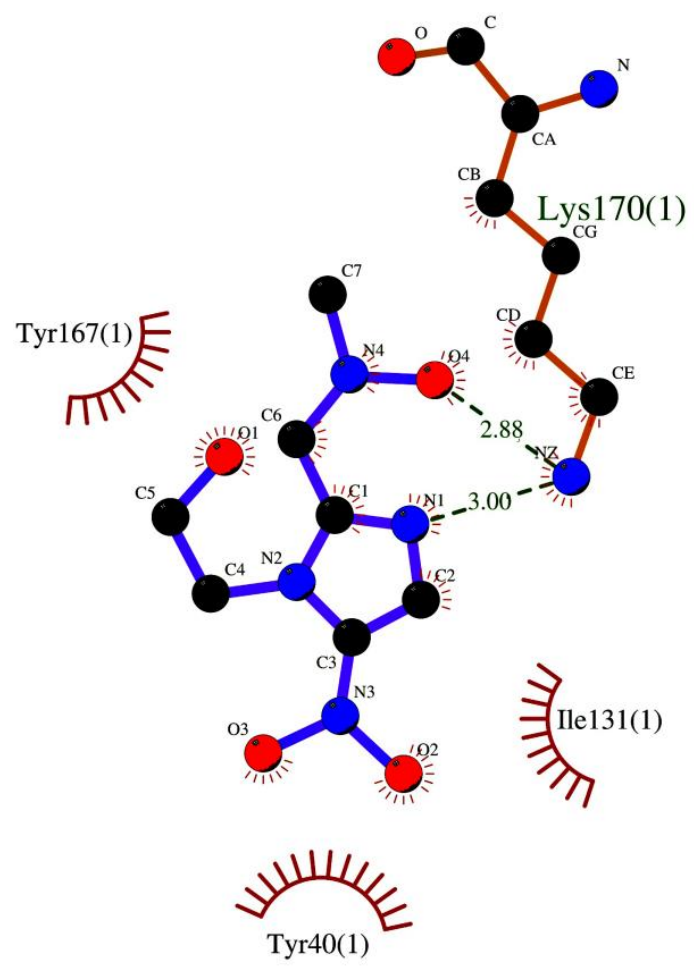

Figure 5. 2D interaction plots of (a) Metronidazole benzoate (b) Metronidazole phosphate and (c) 1-[1-(2Hydroxyethyl)-5-nitroimidazol-2-yl]-N-methylmethanimine oxide 12 (green line: Hydrogen bond and distance between atoms)

Table 3. Hydrogen and hydrophobic binding between selected structure and IL-12

\begin{tabular}{ccc}
\hline Compound & Hydrogen bonding & Hydrophobic binding \\
\hline Metronidazole phosphate (6) & Gln 20,Ser 192,Asp188,Arg & - \\
291, Asp270, Ser 248 & Gln144,Ala176,Glu173,Arg138,Cys177, \\
Metronidazole benzoate (7) & - & Ala180,Glu182,Ser175 \\
$\begin{array}{c}\text { 1-[1-(2-Hydroxyethyl)-5- } \\
\text { nitroimidazol-2-yl]-N- } \\
\text { methylmethanimine oxide }\end{array}$ & Lys170 & Tyr40,Tyr167,Ile131 \\
(13)
\end{tabular}




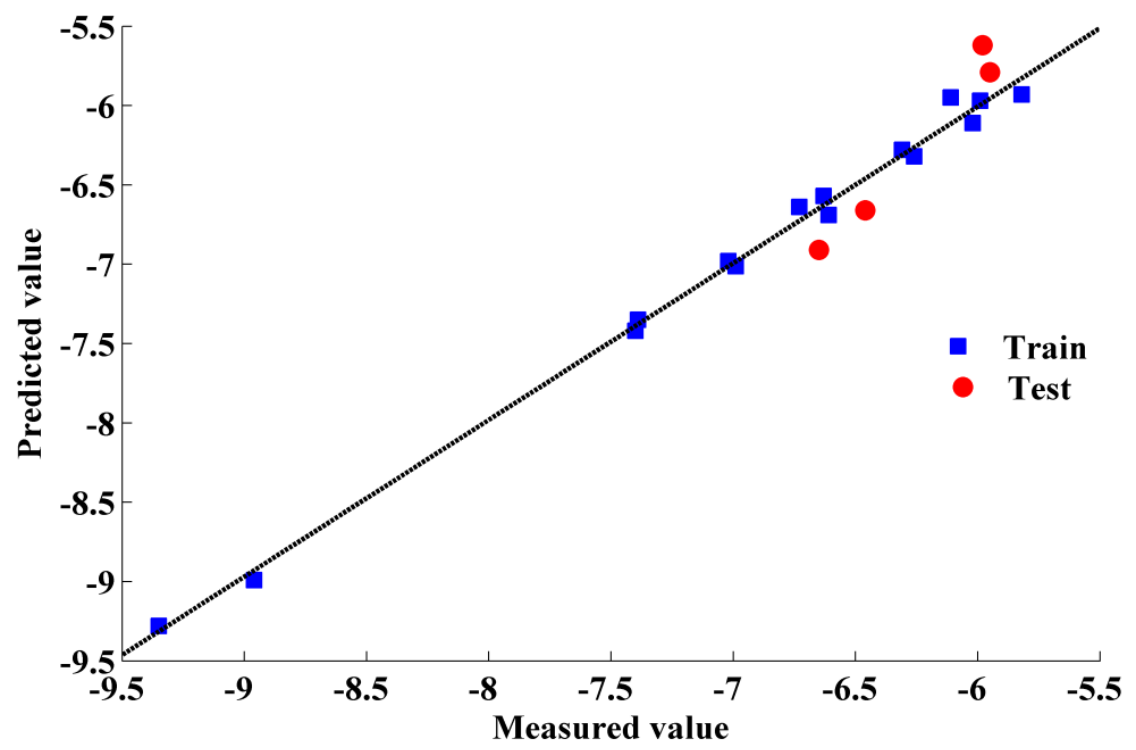

Figure6. Measured and of the predicted value of $\Delta \mathrm{G}$ obtained from QSAR study (Blue: Train data set, Red: Test data set). Root Mean Square Error for train and test data set were 0.06 and 0.123 repectively

Table 4. Pharmacokinetics results of selected compounds obtained from SwissADME

\begin{tabular}{cccc} 
& & & Chemical structure \\
GI absorption & $\mathrm{High}$ & $\mathrm{No}$ & $\mathrm{No}$ \\
BBB permeant & $\mathrm{No}$ & $\mathrm{No}$ & $\mathrm{No}$ \\
P-gp substrate & $\mathrm{No}$ & $\mathrm{No}$ & $\mathrm{No}$ \\
CYP1A2 inhibitor & $\mathrm{No}$ & $\mathrm{No}$ & $\mathrm{No}$ \\
CYP2C19 inhibitor & $\mathrm{No}$ & $\mathrm{No}$ & $\mathrm{No}$ \\
CYP2C9 inhibitor & $\mathrm{No}$ & $\mathrm{No}$ & $\mathrm{No}$ \\
CYP2D6 inhibitor & $\mathrm{No}$ & $-8.89 \mathrm{~cm} / \mathrm{s}$ & $-7.36 \mathrm{~cm} / \mathrm{s}$ \\
CYP3A4 inhibitor & $-8.42 \mathrm{~cm} / \mathrm{s}$ & & \\
Log Kp (skin permeation & & & $\mathrm{No}$ \\
\hline
\end{tabular}


FDA approved drugs was used to find another chemical drug -based on virtual screening of the small molecule from several libraries that gets stable complex by IL-12 to inhibit the activity of this cytokine. In this regard, 13 compounds were selected, and its interaction with interleukin 12 was studied and compared with MTZ results (Figure 7) (Table 5) [18].

Table 5. DrugBank ID, Similarity Score, usual Name and code of different FDA approved drug

\begin{tabular}{|c|c|c|c|c|}
\hline Entry & DrugBank ID & Similarity Score & Usual Name & Code \\
\hline 1 & DB00709 & 0.297 & Lamivudine & $3 \mathrm{TC}$ \\
\hline 2 & DB00879 & 0.252 & Emtricitabine & FTC \\
\hline 3 & DB00900 & 0.217 & Didanosine & DDI \\
\hline 4 & DB00649 & 0.208 & Stavudine & D4T \\
\hline 5 & DB00856 & 0.205 & Chlorphenesin & $\mathrm{CPN}$ \\
\hline 6 & DB00495 & 0.196 & Zidovudine & ZDV \\
\hline 7 & DB00874 & 0.194 & Guaifenesin & Guaif \\
\hline 8 & DB00388 & 0.181 & Phenylephrine & $\mathrm{PN}$ \\
\hline 9 & DB00299 & 0.169 & Penciclovir & PCV \\
\hline 10 & DB00651 & 0.163 & Dyphylline & Dyp \\
\hline 11 & DB00787 & 0.136 & Aciclovir & $\mathrm{ACV}$ \\
\hline 12 & DB00360 & 0.133 & Tetrahydrobiopterin & BH4,THB \\
\hline 13 & DB00446 & 0.132 & Chloramphenicol & $\mathrm{CPN}$ \\
\hline
\end{tabular}




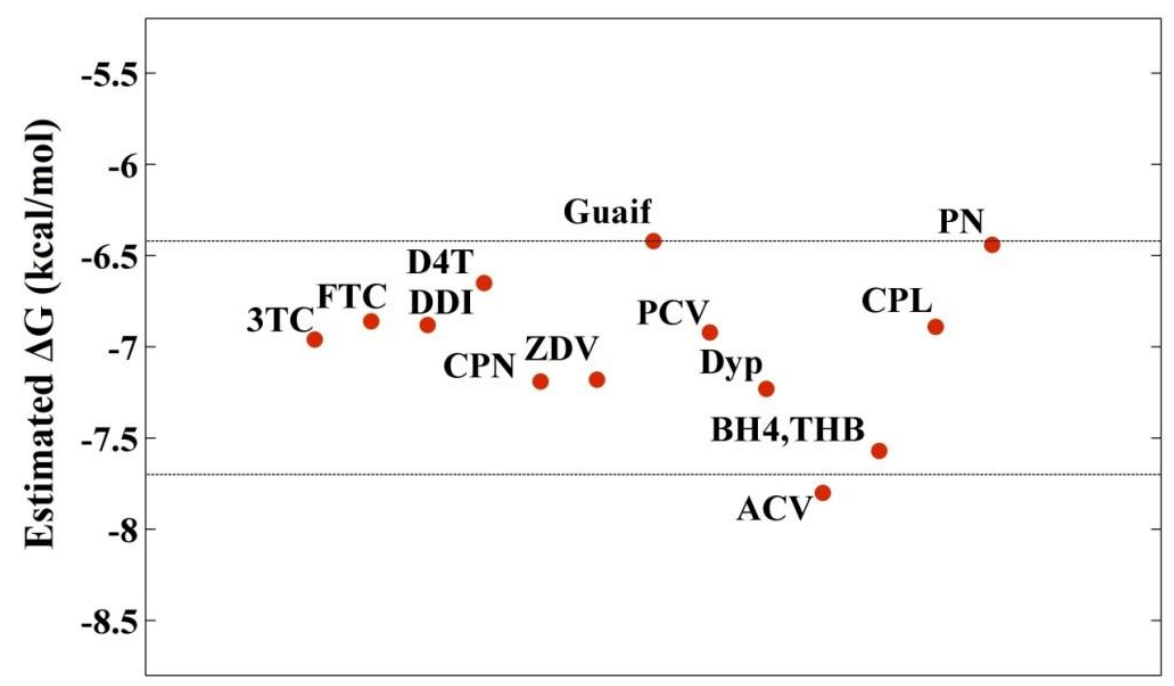

Type

Figure 7. Comparison of estimated $\Delta \mathrm{G}$ of FDA approved drugs which similar to metronidazole. These drugs obtained from swisssimilarity

The results showed that two drugs, acyclovir (ACV), and tetrahydrobiopterin, form more stable complexes with IL-12. ACV is an antiviral drug used to treat viral illnesses, especially herpes simplex. BH4. THB converts several amino acids to other essential molecules in the body, including neurotransmitters. Markkert et al. showed that inhibition of macrophages by acyclovir reduced IL-12 but did not study its impact on IL-12 itself [17]. As well, there is no report about study the effect of tetrahydrobiopterin on IL-12. By comparing the two-dimensional interaction plots of ACV -IL12 and BH4. THB-IL-12, showed that like metronidazole, they have failed any hydrogen bonding to the active site of IL-12 (Figure 8). In addition, the hydrogen and hydrophobic bonds in ACV are greater than BH4. THB. The result of hydrogen and hydrophobic bonds is shown in Table 6.

Table 6. Hydrogen and Hydrophobic bonds of Acyclovir and Tetrahydrobiopterin by IL-12

\begin{tabular}{ccc}
\hline Compound & Hydrogen bond & Hydrophobic bond \\
\hline \multirow{2}{*}{ Acyclovir } & Arg138, Ala179, Thr186, & Ala180, Pro178, Ile207, Tyr114, \\
& Tyr246, Tyr293 & Arg208, Glu182, Arg189, \\
Tetrahydrobiopterin & Glu181, Val185, Ile182 \\
& Asn71, Thr239 & Ser115, Asn113, Ala176, \\
& & Pro178, Ser241, Trp240, Gln172 \\
\hline
\end{tabular}


(a)

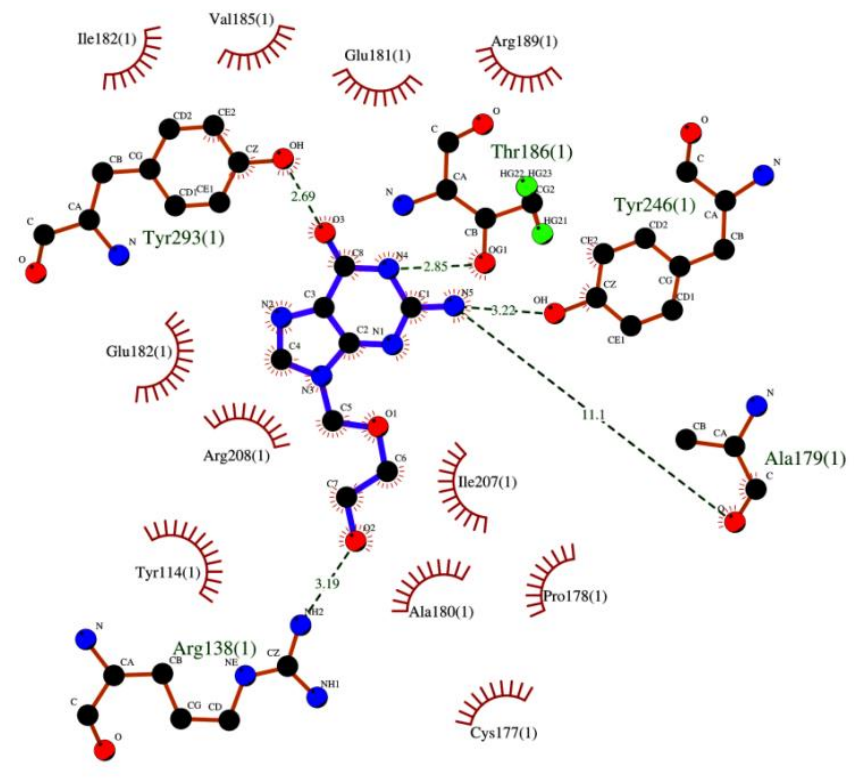

(b)

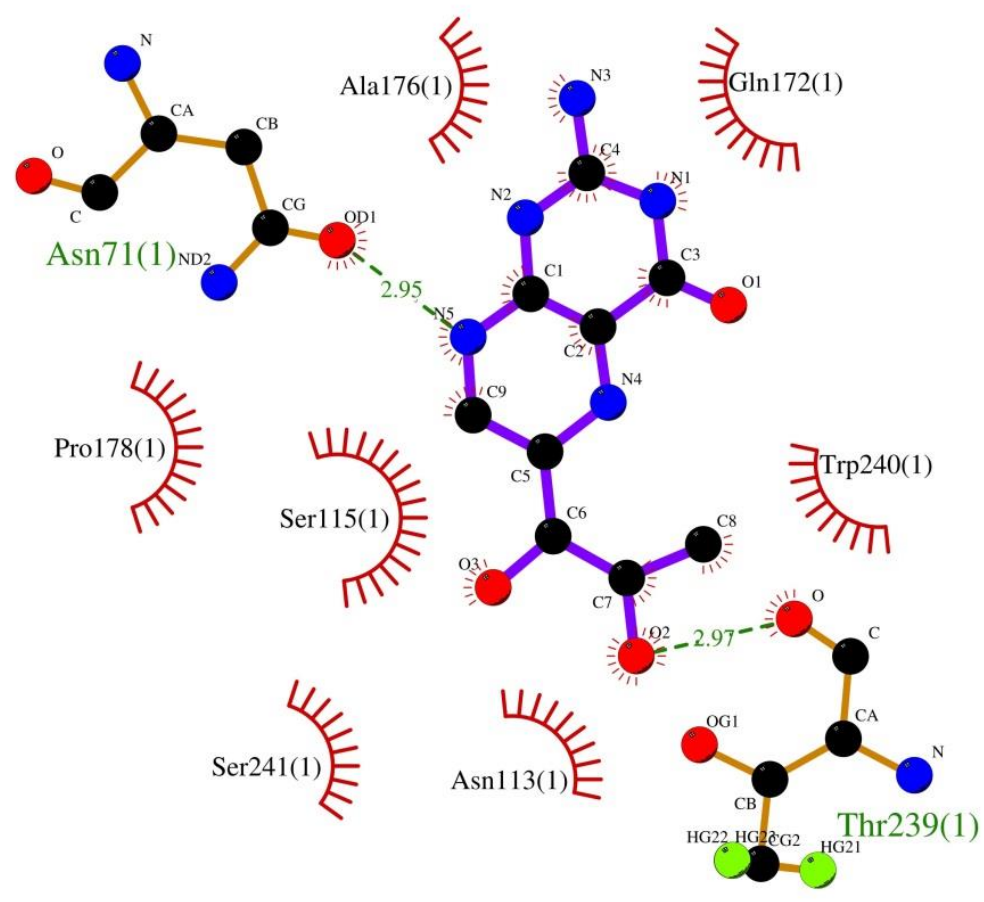

Figure 8. 2D interaction plots of (a) Acyclovir and (b) Tetrahydrobiopterin (green line: Hydrogen bond and distance between atoms) 
The variations of the surface area and volume for IL12-drug complexes were investigated and compared with Apilimod as IL-12 inhibitor (Figure 9). The results revealed that the changes in surface area and volume observed for these complexes are almost identical to the variation detected for Apilimod, moreover, it is suggested that this increase in surface area and volume prevents binding to the receptor.

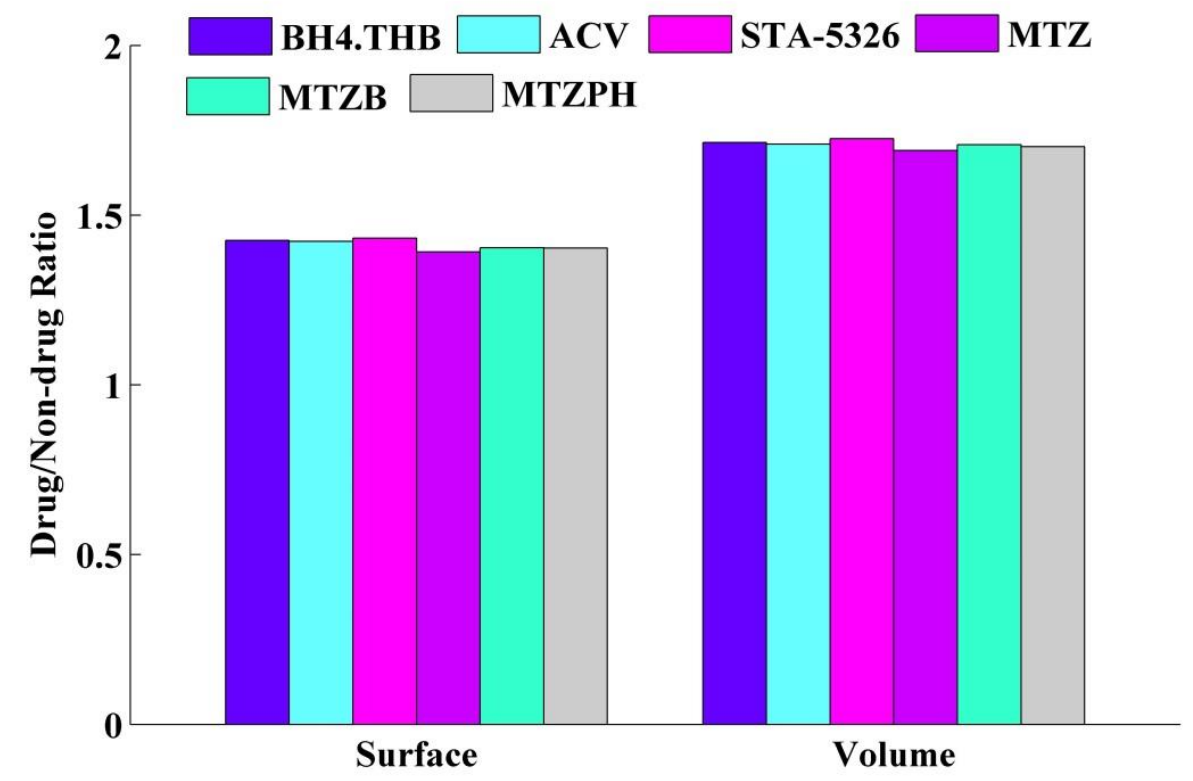

Figure 9. Surface area and volume of IL-12-drug complexes: Tetrahydrobiopterin (BH4.THB) (Blue), Acyclovir (ACV) (light blue). Metronidazole (MTZ) (Purple), Metronidazole benzoate (MTZB) (Cyan), Metronidazole phosphate (MTZPH) (Gray) and control drug Apilimod (STA-5326) (Pink) as IL-12 inhibitor.

The effect of the distance between oxygen and the importance of the hydroxyl group on inhibition was studied. The results showed that increasing the distance caused to increase the stability of the complex and inhibits the active site of protein by getting hydrogen bonds with Ser183 amino acid (Figure 10). Changing the position of hydroxyl and methyl functional group leads to the binding with the active site of IL-12, through hydrogen bonding with Ser183, Lue184 and Glu 173 with almost the same binding energy.

(a) 


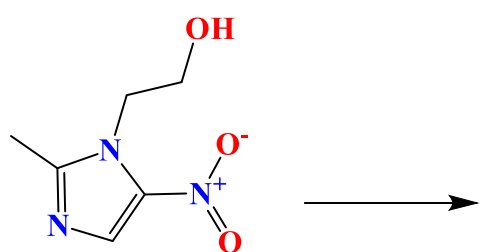

Metronidazole

Estimated G $(\mathrm{kcal} / \mathrm{mol})=-6.4$

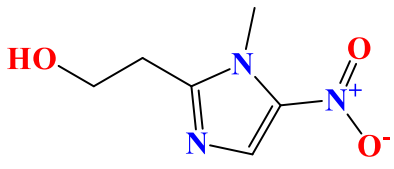

1-Methyl-5-nitroimidazole-2-ethanol

Estimated G $(\mathrm{kcal} / \mathrm{mol})=-6.55$

(b)

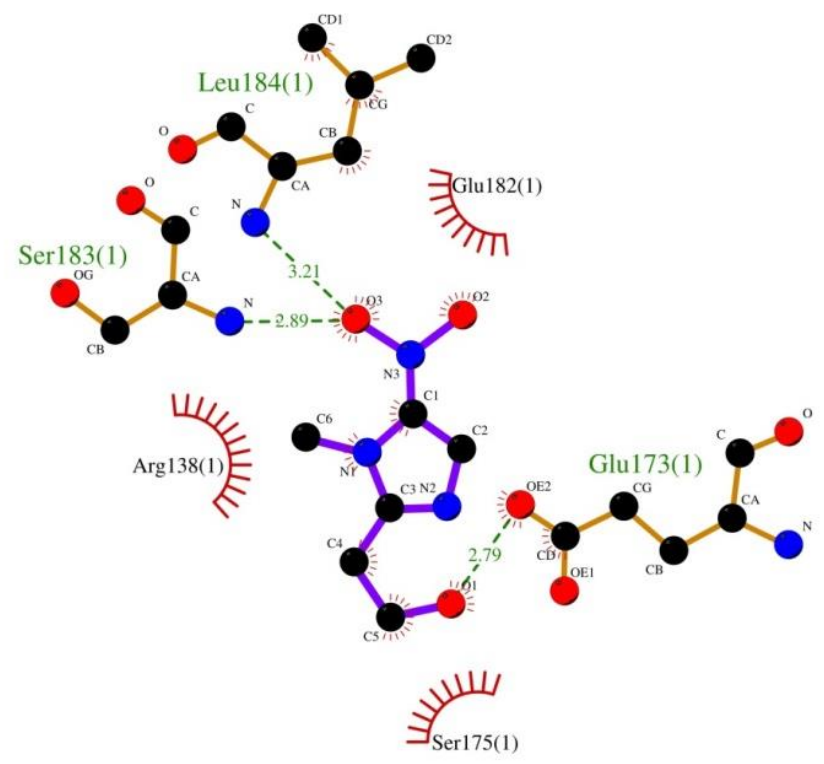

Figure 10. a) Comparison of chemical structure and estimated $\Delta \mathrm{G} \mathrm{b}$ ) 2d 1-Methyl-5-nitroimidazole-2ethanol -IL-12 interaction plot (green line shows the hydrogen bond and distance)

\section{Conclusion}

The present study shows that IL-12 is a significant inflammatory marker that may help clinicians identify severe COVID-19 patients in the early stage of the disease. By modifying the surface area and volume of IL-12, MTZ prevents IL-12 from binding to the IL-12 receptors. Virtual screening of certain other drugs approved by the U.S. Food and Drug Administration (FDA), such as metronidazole phosphate, metronidazole benzoate, acyclovir, tetrahydrobiopterin, and 1[1- (2- hydroxyethyl) -5-nitroimidazole-2-yl] - N-methylmethanimine oxide and the study of their interactions with IL-12 revealed that, like MTZ, they prevent the binding of IL-12 to receptors by modifying the surface and the volume. Also, altering the position of methyl and hydroxyl functional groups in MTZ triggered the inhibition of the active sites of IL-12. This 
report serves as a symbol of a slight step in international cooperation to help human society solve the global health problem.

\section{Reference}

1. Ng M-Y, Lee EY, Yang J, Yang F, Li X, Wang H, et al. Imaging profile of the COVID-19 infection: radiologic findings and literature review. Radiology: Cardiothoracic Imaging. 2020;2(1):e200034.

2. Mehta P, McAuley DF, Brown M, Sanchez E, Tattersall RS, Manson JJ, et al. COVID-19: consider cytokine storm syndromes and immunosuppression. Lancet (London, England). 2020;395(10229):1033.

3. Singhal T. A review of coronavirus disease-2019 (COVID-19). The Indian Journal of Pediatrics. 2020:1-6.

4. Lazzerini PE, Boutjdir M, Capecchi PL. COVID-19, arrhythmic risk and inflammation: mind the gap! Circulation. 2020.

5. Pacha O, Sallman MA, Evans SE. COVID-19: a case for inhibiting IL-17? Nature Reviews Immunology. 2020;20(6):345-6.

6. Brogden R, Heel R, Speight T, Avery G. Metronidazole in anaerobic infections: a review of its activity, pharmacokinetics and therapeutic use. Drugs. 1978;16(5):387-417.

7. Gharebaghi R, Heidary F, Moradi M, Parvizi M. Metronidazole; a Potential Novel Addition to the COVID-19 Treatment Regimen. Archives of Academic Emergency Medicine. 2020;8(1).

8. Jorgensen WL. The many roles of computation in drug discovery. Science. 2004;303(5665):1813-

8.

9. Winkler DA. The role of quantitative structure-activity relationships (QSAR) in biomolecular discovery. Briefings in bioinformatics. 2002;3(1):73-86.

10. Wallace AC, Laskowski RA, Thornton JM. LIGPLOT: a program to generate schematic diagrams of protein-ligand interactions. Protein engineering, design and selection. 1995;8(2):127-34.

11. Binkowski TA, Naghibzadeh S, Liang J. CASTp: computed atlas of surface topography of proteins. Nucleic acids research. 2003;31(13):3352-5.

12. Mauri A, Consonni V, Pavan M, Todeschini R. Dragon software: An easy approach to molecular descriptor calculations. Match. 2006;56(2):237-48.

13. Daina A, Michielin O, Zoete V. SwissADME: a free web tool to evaluate pharmacokinetics, drug-likeness and medicinal chemistry friendliness of small molecules. Scientific reports. 2017;7:42717.

14. Wojno EDT, Hunter CA, Stumhofer JS. The immunobiology of the interleukin-12 family: room for discovery. Immunity. 2019;50(4):851-70.

15. Zundler S, Neurath MF. Interleukin-12: Functional activities and implications for disease. Cytokine \& growth factor reviews. 2015;26(5):559-68.

16. Liu J, Cao S, Kim S, Chung EY, Homma Y, Guan X, et al. Interleukin-12: an update on its immunological activities, signaling and regulation of gene expression. Current immunology reviews. 2005;1(2):119-37.

17. Markert JM, Cody JJ, Parker JN, Coleman JM, Price KH, Kern ER, et al. Preclinical evaluation of a genetically engineered herpes simplex virus expressing interleukin-12. Journal of virology. 2012;86(9):5304-13.

18. Zoete V, Daina A, Bovigny C, Michielin O. SwissSimilarity: a web tool for low to ultra high throughput ligand-based virtual screening. ACS Publications; 2016. 